\title{
REALITY CAPTURE AND VISUALISATION OF 3D OIL \& GAS FACILITY DATA FOR OPERATIONS AND DECOMMISSIONING
}

\author{
João Patacas ${ }^{1}$, Huda Dawood ${ }^{2}$, and Nashwan Dawood ${ }^{3}$
}

\begin{abstract}
Accurate and reliable data is fundamental for the management of Oil \& Gas facilities throughout their lifecycle. Several standards and specifications have been proposed throughout the years to support information management in the Oil \& Gas industry. Standards such as ISO 15926 and specifications such as Capital Facilities Information Handover Specification (CFIHOS) are slowly being adopted by the Oil \& Gas industry in new build projects. However, for existing facilities in the Operations and Decommissioning stages, information is frequently of poor quality. This study proposes the visualisation of 3D Oil \& Gas asset data, including documentation data from several databases based on CFIHOS templates, in order to support the planning of Operations and Decommissioning tasks. We propose the use of game engines for the visualisation of the data-enriched 3D models. The proposed method supports the input of 3D data from reality capture methods, as well as existing design and as-built facility models. The proposed method was tested in a case study of an existing Normally Unmanned Installation (NUI) using data from a laser scanning survey as an input. Several limitations were highlighted and future developments have been identified, including the deployment of the virtual environment on mobile platforms.
\end{abstract}

Keywords: Reality capture, Oil \& Gas facilities, CFIHOS, ISO 15926, serious games.

\section{INTRODUCTION}

Oil and Gas projects have a unique set of characteristics: they are typically large-scale projects with high complexity, high volumes of design and real-time data, and high risk levels due to their hazardous nature (Hou et al. 2014). Information management in Oil and Gas projects is fundamental for their success, however it entails various challenges due to the size and complexity of the facilities. Information in Oil and Gas projects is typically generated by different project stakeholders throughout the lifecycle of facilities and stored across various databases and documents (Rasys et al. 2014). As project complexity increases, it becomes increasingly difficult to manage such an array of inter-related documents. In particular, spatial relations between different components become increasingly hard to follow (Hou et al. 2014), creating problems during the latter stages of the Oil \& Gas assets lifecycle, i.e. Operations and Decommissioning.

The Operations and Decommissioning stages of Oil and Gas facilities lifecycle are highly dependent on accurate data, in order to support decisions on assets and their components. For decommissioning this includes the possibilities for: a) reuse - a possibility for floating platforms and Normally Unmanned Installations (NUI) which make up $20 \%$ of the total offshore assets, b) Scrap and c) Repurposing existing assets e.g. Cranes and Gas Turbines in other industries or as spare parts.

KTP Associate, Technology Futures Institute, Teesside University, UK, j.patacas@tees.ac.uk

Technology Futures Institute, Teesside University, UK, h.dawood@tees.ac.uk

Professor, Technology Futures Institute, Teesside University, UK, n.n.dawood@tees.ac.uk 
The use of engineering data management standards and specifications has been proposed to streamline the access and delivery of data throughout the lifecycle of Oil \& Gas assets (USPI-NL 2011, Rasys et al. 2012, Fiatech 2017a, Fiatech 2017b). The ISO 15926 standard provides a common language to enable the integration, sharing, exchange, and handover of data about process industry assets, including Oil \& Gas assets, between disparate computer systems (ISO 2004). Several projects based on this standard are currently under development to enable its adoption by the Oil \& gas sector (USPI-NL 2011, Fiatech 2017a, Fiatech 2017b). The Capital Facilities Information Handover Specification (CFIHOS) project, currently under development, will provide a practical implementation of the ISO 15926 standard. CFIHOS proposes the definition of several information management specification templates to support the whole lifecycle of process plants, including the specification of a Reference Data Library, which specifies the required documentation at each phase of the assets' lifecycle (USPI-NL 2011). Fiatech has developed guides as well as various tools for the implementation of the ISO 15926 standard by the Oil \& Gas industry, including the development of information patterns for ISO 15926 to enable standardized information access and data exchange between software applications (Fiatech 2017a, Fiatech 2017b).

Oil and Gas facilities are difficult, dangerous and expensive to access. The visualisation of Oil \& Gas assets has been the focus of recent research studies (Rasys et al. 2014, Hou et al. 2014, Wang et al. 2014). Visualisation tools could enable Oil and Gas consultants and clients to access metadata enhanced 3D models of assets, improving the planning of maintenance and decommissioning procedures offshore, while reducing associated costs and risks. While data integration can enable decision making in operations and decommissioning procedures, 3D models are not suitable to store all the attribute data required for Oil \& Gas facilities due to the size of facilities and amount of data required (Rasys et al. 2014). Furthermore, while 3D design and/or as-built models might be available for new facilities, such data will not be available for facilities at the end of their lifecycle for decommissioning. Hence, a methodology is necessary to enable the capture of 3D data offshore and linking this data to asset documentation data stored in disparate systems and databases.

The underlying assumption in this research is that the combined use of standards and specifications for engineering data management, reality capture methods, and visualisation of 3D Oil and Gas assets, can improve the efficiency of Operations and Decommissioning procedures while reducing their risks and costs. In this research a method is proposed for the capture and visualisation of 3D Oil and Gas assets along with their associated engineering data. The method proposes the use of the CFIHOS specification for the definition of document-based databases and the integration and visualisation of 3D models with asset data using a game engine. A case study of an existing NUI is developed to demonstrate the proposed method, including reality capture through a laser scanning survey.

\section{PRoposed METHOD}

In this paper, a method is proposed to enable the integration of 3D Oil and Gas facility data with asset data from document databases based on CFIHOS templates, in order to provide accurate data for the execution of tasks during operations and decommissioning of facilities. The method proposed in this study is based on the authors' on going research in the visualisation of building assets' data for facilities management (Patacas et al. 2016). 3D data for Oil \& Gas facilities can be obtained from as-built models, modelled from as- 
built drawings, or it can be obtained using reality capture methods such as laser scanning or photogrammetry.

To support operations and decommissioning projects for facilities approaching the end of their lifecycle, 3D data will typically not be available. A large number of Oil and Gas installations in the North Sea are coming to the end of their life and it is expected that over the next couple of decades many of these facilities will be taken out of service and decommissioned (USPI-NL 2011). Given the age of these facilities, 3D data and drawings are typically not available. Therefore it is fundamental to investigate how reality capture methods can be used effectively to obtain 3D data for these facilities to support operations and decommissioning procedures. The use of reality capture methods can also provide valuable inputs regarding assets' condition. Since both laser scanning and photogrammetry technology can provide detailed geometry as well as colour, they can be used to identify corrosion and other defects in Oil \& Gas assets.

The proposed method supports the use of 3D data from existing design/as-built models, along with data obtained from reality capture methods such as laser scanning and photogrammetry. Requirements for the use of reality capture data include cleaning and optimisation of data sets before they can be converted to a 3D model. Another key requirement is the adoption of a consistent Tag naming convention for the assets, which will be used to establish the link between 3D objects and corresponding Tag objects within the project's document database. Tag naming procedures are typically defined at the start of Oil \& Gas projects and should be adopted consistently throughout the development of documentation databases and 3D models to enable the creation of automated links between them.

We propose the use of game engines to provide the visualisation of 3D Oil and Gas facility models and their integration with asset data from documentation databases. The use of Serious Games and Virtual Reality for the training of construction workers has been proposed in various studies in the Architecture, Engineering and Construction (AEC) sector (Lin et al. 2011; May et al. 2012; Addison et al. 2013; Chen et al. 2013; Chi et al. 2013; Dawood et al. 2014; Chang 2015), and it is expected that the benefits from these applications can also be realised in the Oil and Gas sector (Rasys et al. 2014, Hou et al. 2014, Wang et al. 2014). 3D models of Oil and Gas facilities - obtained from design/asbuilt models or drawings, or through reality capture methods - can be optimised and imported into a game engine for visualisation. The user can interact with the virtual environment and retrieve the relevant assets' data from the facility's document databases. The game engine establishes a connection to an external server, which retrieves the relevant data from the documents database(s), and passes the data back to the game engine where it can be displayed upon interaction with the 3D model. The proposed method is summarised in Fig. 1. In the following section the proposed method will be applied to a case study of an existing NUI. 


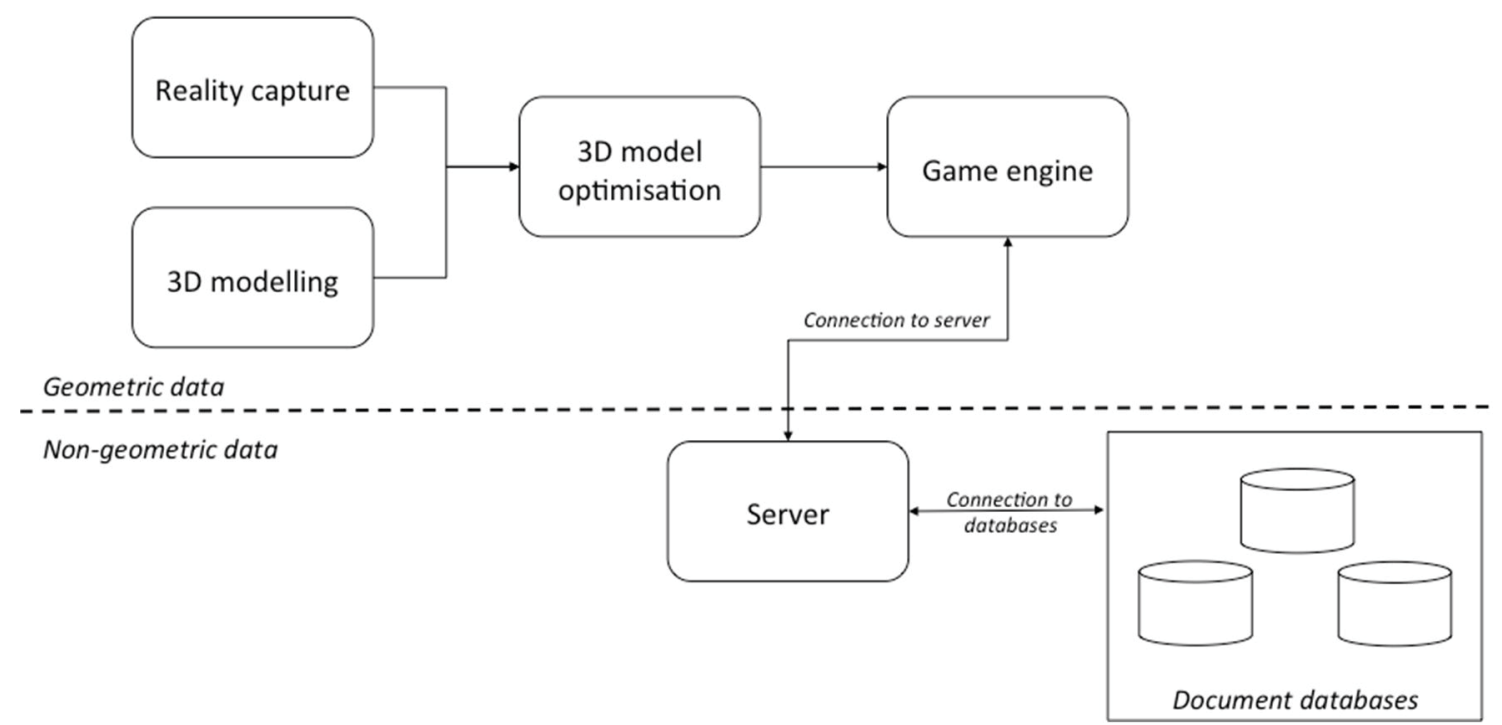

Figure 1: Method for the integration of 3D Oil and Gas facility data with asset data from document databases

\section{CASE STUDY}

The method proposed in this study was applied to an existing NUI. Since a 3D model of the facility was not available, a laser scanning survey was performed onsite to capture the NUI's geometry.

Despite its relatively small size (approximately $78 \mathrm{~m}^{2}$ of floor area, and $20 \mathrm{~m}$ height above sea level), due to the complexity of the NUI's geometry, a total of 47 laser scans were performed to accurately capture the facility. Following this process, a 3D model was generated, optimised and imported into the Unity game engine where individual assets were linked to the corresponding Tag objects within the NUI's underlying documentation database. The following steps, which are summarised in Fig. 2, were performed in order to provide the visualisation of the NUI using reality capture data, along with its related documentation data:

1. Import laser scanning survey data and alignment of laser scans using Autodesk Recap Pro.

2. Generation of point cloud from survey data and point cloud clean up.

3. Definition of view states and generation of individual meshes using Recap Pro, according to the document database Tag definitions.

4. Import individual mesh files into Autodesk 3DS Max software.

5. Optimisation of meshes within 3DS Max software.

6. Export geometric model as an FBX file from 3DS Max. The model is exported as separate geometric objects (as opposed to a single mesh) to allow mapping to individual Tags within the documentation database.

7. Set up a new scene in the Unity game engine and import the FBX file. This includes assignment of mesh colliders to the model's game objects. Given the complexity of models' meshes (even after optimisation) it is recommended to assign colliders manually to improve in-game performance. 
8. Checking and correcting import errors. Errors that were experienced by the authors included errors in texture assignments. These issues were fixed within Unity.

9. Retrieve data from documents database through the definition of methods in a Node.js server.

10. Link individual game objects to the external asset documents database using Socket.IO for the definition of scripts in Unity.

To display facility Tag data on-screen, scripts were defined within the Unity game engine to react to users' interactions with the model. In order to display assets' Tag data on-screen, colliders are defined and associated to the corresponding game objects. When a user clicks on object with associated Tag data, a connection is established to the asset documents database and the corresponding Tag data is retrieved and displayed on screen. Fig. 3 shows part of the point cloud model obtained from the laser scanning survey along with the 3D model and associated Tag data in Unity.

Access to the external asset documents database is provided through the Socket.IO library. Socket.IO is a JavaScript library that enables real time, bi-directional communication between web clients and servers (Socket.IO, 2017). In this case study the Socket.IO extension for Unity was imported into the project and used to establish the connection between Unity and a Node.js server. The Node.js server then connects to a MongoDB database and retrieves the asset documents data. The use of semi-structured data storage such as MongoDB has been proposed to improve read access of document databases for large facilities, when compared to relational databases such as MySQL (Rasys et al. 2012).

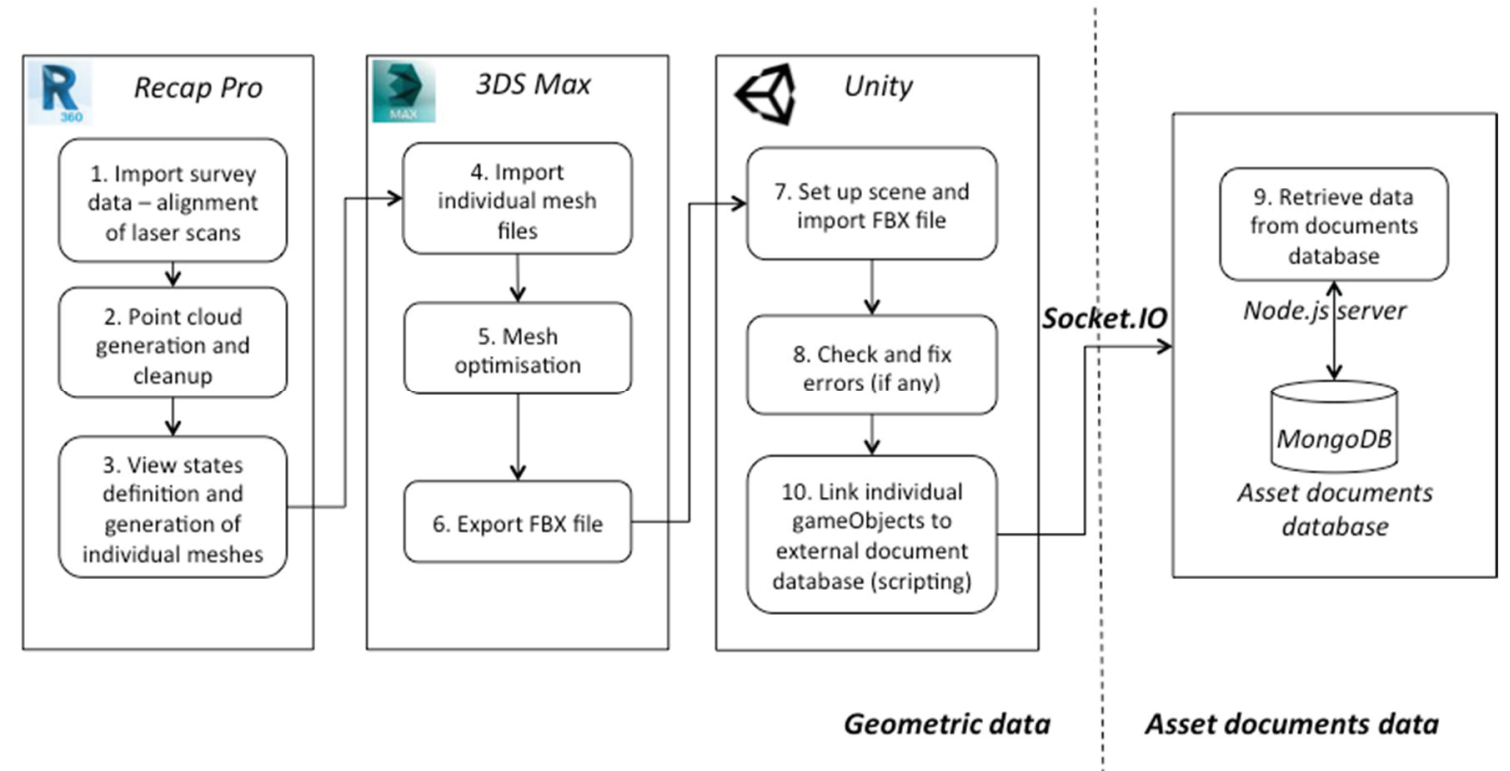

Figure 2: Workflow for the visualisation of the 3D Oil and Gas facility with associated documentation data in the Unity game engine 

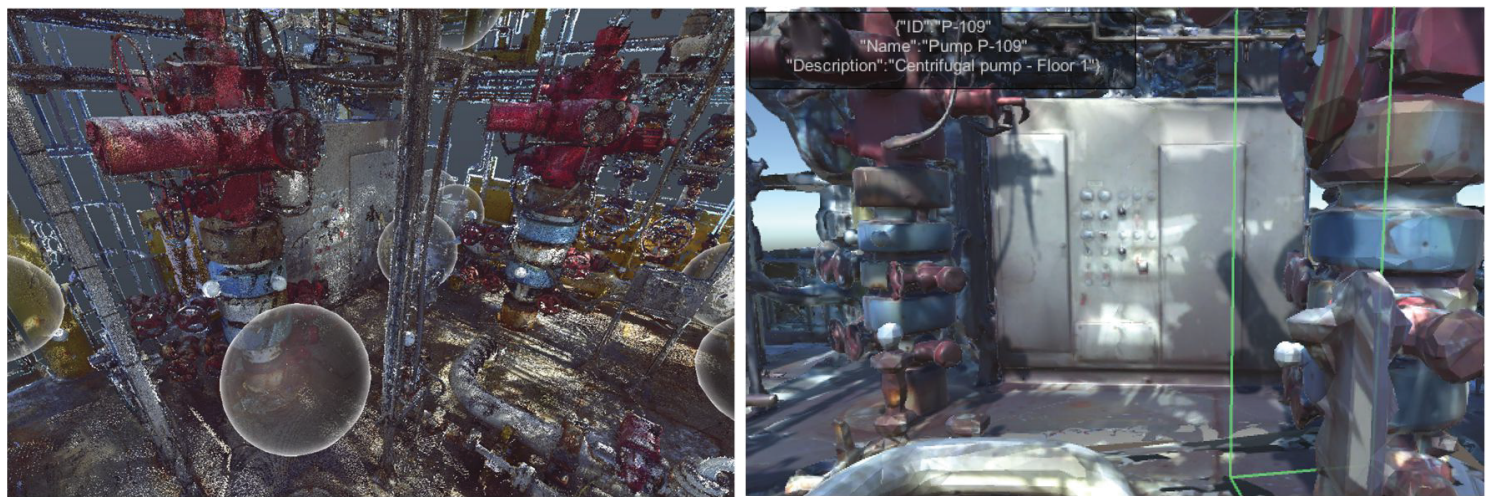

Figure 3: Point cloud model obtained from laser scanning survey in Recap Pro (left); Displaying 3D model and associated Tag data from an asset in Unity (right).

\section{DISCUSSION}

The implementation of the proposed method within this case study has highlighted a number of challenges:

1) Data set size and geometry complexity: The NUI facility is small when compared to typical Oil \& Gas facilities. However, due to its complex geometry, a high number of laser scans had to be performed, which resulted in a heavy point cloud model (around 3GB). Mesh conversion of this model resulted in extremely complex meshes which had to be optimised using the 'ProOptimizer' batch tool in 3DS Max, in order to obtain an acceptable user experience in the virtual environment.

2) Errors in texture assignments: One of the advantages of using reality capture methods such as laser scanning and photogrammetry is obtaining texture data which can provide information about the assets condition. Upon import of the mesh model into the Unity game engine, textures had to be reassigned to the individual game objects. For larger facilities with many assets this issue can introduce large amounts of rework.

Both of these challenges can undermine the application of the proposed method for large facilities and more research is needed regarding the optimisation of the 3D model. The use of a laser scanner for data capture provides a dimensionally accurate point cloud, however, the conversion of point cloud to mesh and subsequent mesh optimisation procedures result in a loss of dimensional accuracy. This loss of dimensional accuracy is needed in order to increase the performance of the virtual environment and provide a good experience to the end user. The use of an external server to access the documentation database also improves the virtual environment's performance, since database access is provided through the definition of methods in the server. This approach provides a layer of security, compared to direct database access from the game engine. It also enables the use of the proposed method with other databases such as MySQL or PostgreSQL.

The adoption of the proposed method is expected to reduce costs and risks associated with operations and decommissioning procedures in Oil \& Gas facilities. A game engine was used for the development of the virtual environment since the interactive visualisation 
it provides enables a more immersive approach when compared to design review applications. The virtual model can be used to locate assets and their associated documents providing key inputs to the planning of operations and decommissioning procedures before going offshore, saving valuable time and costs in offshore man-hours and reducing risks associated with the execution of maintenance tasks.

The use of Unity and other game engines also allows deployment on several platforms including web-based and mobile platforms, as opposed to design review/visualisation software which are typically only available on desktop computers. Moreover, even though a selection of specific tools was used for the case study, the proposed method could be applied with other similar tools from different providers as well.

\section{CONCLUSIONS}

This research proposed a method to provide the visualisation of 3D Oil and Gas facilities and their associated documentation data using a game engine. The proposed approach was applied to a case study of an existing NUI facility with the goal of demonstrating how documentation data can be integrated with 3D models and used for the planning of operations and decommissioning procedures. The 3D data for the facility was obtained using laser scanning to obtain a point cloud model, which was subsequently converted into meshes and optimised. This process revealed limitations related to dimensional accuracy and to the virtual environment's performance. Despite these limitations, it was possible to visualise the model's assets and selectively retrieve their associated documentation data using the virtual environment. The proposed method enables users to interactively navigate the model and retrieve relevant Tag data about assets, including asset installation and decommissioning procedures.

Future developments will address the virtual environment's performance issues in order to enable the application of the proposed method to large Oil \& Gas facilities, and to enable the deployment of the virtual environment on mobile platforms.

\section{REFERENCES}

Addison, A., O’Hare, W.T., Kassem, M. and Dawood, N. (2013). The importance of engaging engineering and construction learners in virtual worlds and serious games, CONVR 2013. London, UK, 30-31 October, pp. 448- 457.

Chang, D. (2015). Total Facilities Management Simulations. Available at: http://www.tribe-studios.com/blog/total_facilities_management_simulations [Accessed: 14/01/2017]

Chen, A., Golparvar-Fard, M. and Kleiner, B. (2013). SAVES: A safety training augmented virtuality environment for construction hazard recognition and severity identification, CONVR 2013. London, October 30- 31.

Chi, H., Kang, S. and Wang, X. (2013). Research trends and opportunities of augmented reality applications in architecture, engineering, and construction, Automation in Construction, 33(0), pp. 116-122.

Dawood N., Miller G., Patacas J. and Kassem M. (2014). Construction health and safety training: the utilisation of $4 \mathrm{D}$ enabled serious games, ITcon Vol. 19, Special Issue BIM Cloud-Based Technology in the AEC Sector: Present Status and Future Trends, pg. 326-335, http://www.itcon.org/2014/19

Decom North Sea. (2017). Decommissioning Explained. Available at: http://decomnorthsea.com/decom-explained [Accessed: 14/01/2017] 
Fiatech. (2017a). ISO 15926 Information Models and Proteus Mappings (IIMM) Available at: $\quad$ http://www.fiatech.org/design/projects/589-iringtools-interfacing-project [Accessed: 14/01/2017].

Fiatech. (2017b). ISO 15926 Information Patterns (IIP) Available at: http://www.fiatech.org/information-management/projects/1161-iso-15926information-models-and-proteus-mappings-iimm [Accessed: 14/01/2017].

Hou, L., Wang Y., Wang X., Maynard M., Cameron I., Zhang S. and Jiao I. (2014). Combining Photogrammetry and Augmented Reality Towards an Integrated Facility Management System for the Oil Industry, Proceedings of the IEEE. pp. 204-220.

ISO (2004) ISO 15926-1:2004 - Industrial automation systems and integration -Integration of life-cycle data for process plants including oil and gas production facilities -- Part 1: Overview and fundamental principles. International Organisation for Standardisation, Geneva, Switzerland.

Lin, K., Son, J.W. and Rojas, E.M. (2011). A pilot study of a 3D game environment for construction safety education, ITCon, 16(Special Issue Use of Gaming Technology in Architecture, Engineering and Construction), pp. 69-84.

May, M., Holzer, F. and Otto, F. (2012). eLearning in Facility Management by Serious Games, Proceedings of the 7th International Conference on eLearning. The Chinese University of Hong Kong, 21-22 June 2012, pp. 294-304.

Patacas, J., Dawood, N., Greenwood, D., and Kassem, M. (2016). Supporting building owners and facility managers in the validation and visualisation of asset information models (AIM) through open standards and open technologies, ITCon, 21(Special issue CIB W78 2015 Special track on Compliance Checking), pp. 434-455.

Rasys, E., Hodds, M., Dawood, N. and Kassem, M. (2012). A Web3D Enabled Information Integration Framework for Facility Management, Australasian Journal of Construction Economics and Building - Conference Series, Vol.2, No 1.

Rasys, E., Dawood, N., Scott, D. and Kassem, M. (2014). Dynamic Web3D Visualisation of Oil \& Gas Facility Assets, Proceedings of the 14th International Conference on Construction Applications of Virtual Reality, pp. 278-288.

Socket.IO (2017) Socket.IO Available at: http://socket.io/ [Accessed: 14/01/2017].

USPI-NL (2011) CFIHOS (Capital Facilities Information HandOver Specification) for process industries. Available ht: ht//uspiglobal.org/index.php/projects/frameworks-methodologies/136-cfihos $\quad$ [Accessed: 14/01/2017].

Wang, X., Truijens, M., Hou, L., Wang, Y. and Zhou, Y. (2014). Integrating augmented reality with building information modeling: Onsite construction process controlling for liquefied natural gas industry, Automation in Construction, 40, pp. 96-105. 